\title{
Impacto de la mediación de la TIC durante la pandemia del covid-19 en la práctica pedagógica de estudiantes de un programa de formación de maestros en matemática
}

\section{Impact of ICT mediation during the covid-19 pandemic on the pedagogical practice of students of a mathematics teacher training program}

\author{
Olga Lucy Rincón Leal ${ }^{1}$ \\ César Augusto Hernández Suárez²
}

Raúl Prada Núñez ${ }^{3}$

UFPS

\footnotetext{
$1 \quad$ Magister en Educación Matemática - Universidad Nacional Experimental del Táchira (Venezuela). Docente investigador de la Universidad Francisco de Paula Santander. Correo electrónico: olgarincon@ufps.edu.co. Orcid: 00000002-8080-496X.

$2 \quad$ Magister en Enseñanza de las Ciencias - Universidad Nacional Experimental del Táchira (Venezuela). Docente investigador de la Universidad Francisco de Paula Santander. Correo electrónico: cesaraugusto@ufps.edu.co. Orcid: 0000-0002-7974-5560.

3 Magíster en Ingeniería de Análisis de Datos, Mejora de Procesos y Toma de Decisiones - Universidad Politécnica de Valencia (España). Docente investigador de la Universidad Francisco de Paula Santander. Correo electrónico: raulprada@ufps.edu.co. Orcid: 0000-0001-61451786.
}

\section{RESUMEN}

El objetivo de este estudio fue describir el desarrollo de los procesos de práctica pedagógica durante la emergencia de la pandemia, e identificar experiencias educativas y aportar elementos de autoevaluación para el desarrollo de las mismas. Se enmarca en las características del enfoque cuantitativo a nivel descriptivo y de corte transversal. 
Para la recolección de los datos se diseñó un cuestionario ad hoc compuesto por 28 ítems con opciones de respuesta cerrada, en los que se pretendía determinar el impacto del proceso adelantado durante los dos semestres del 2020 en la línea de asignaturas de Práctica Pedagógica, en un programa de Licenciatura en Matemáticas. El cierre de las instituciones educativas en todos los niveles educativos, por la pandemia del Covid-19, obligó a crear nuevas formas de interacción entre docentes, estudiantes y comunidad educativa en general, lo que condicionó el desarrollo de las prácticas pedagógicas de forma presencial, pero posibilitó otras formas para continuar con el desarrollo de estos cursos, con el diseño e implementación de estrategias para el acompañamiento de los docentes en formación apoyado especialmente en la mediación de TIC mediante la educación remota.

\section{PALABRAS CLAVE:}

Covid-19, Practica Pedagógica, Docente en formación, mediación, TIC.

\section{SUMMARY}

The objective of this study was to describe the development of pedagogical practice processes during the emergency of the pandemic, and to identify educational experiences and provide elements of self-evaluation for their development. It is framed within the characteristics of the quantitative approach at a descriptive and crosssectional level. For data collection, an ad hoc questionnaire composed of 28 items with closed response options was designed to determine the impact of the process carried out during the two semesters of 2020 in the Pedagogical Practice subject line, in a bachelor's degree program in Mathematics. The closure of educational institutions at all educational levels, due to the Covid-19 pandemic, forced to create new forms of interaction between teachers, students and the educational community in general, which conditioned the development of pedagogical practices in person, but made possible other ways to continue with the development of these courses, with the design and implementation of strategies for the accompaniment of teachers in training supported especially in the mediation of ICT through remote education.

\section{KEYWORDS:}

Covid-19, Pedagogical Practice, Teacher in training, mediation, ICT

\section{INTRODUCCIÓN}

El mundo, actualmente se encuentra atravesando una crisis pandémica del virus Covid-19, que incluye el confinamiento y asilamiento social, lo que ha ocasionado cambios en hábitos y prácticas de las personas, con afectaciones principalmente negativas: depresivas, (GamboaSuárez et al., 2020); fisiológicas (Prada-Núñez, et al., 2020); ocupacionales y emocionales, (Hernández-Vergel et al., 2021); económicas (Barrutia-Barreto et al., 2021); educativas (Ayala-García et al., 2020), entre otras.

A nivel educativo, se han suspendido las actividades académicas presenciales y uno de los mayores retos de las instituciones en todos los niveles educativos, para garantizar la continuidad del proceso educativo, ha sido la transformación y migración abrupta hacia un modelo de educación remota mediada por TIC. Con el aislamiento de los actores del proceso educativo, ha surgido una serie de efectos múltiples, especialmente en los docentes y en los estudiantes (personales, económicas, salud, afectivas, entre otras).

En el caso de las instituciones de educación superior, para cumplir con las disposiciones gubernamentales (Gobierno de Colombia Ministerio de Educación Nacional), han tomado medidas para proporcionar (en la medida de sus posibilidades) a docentes y estudiantes infraestructura tecnológica para continuar 
desarrollando sus actividades académicas. Esta transición de la modalidad presencial a la modalidad remota asistida con recursos TIC obligó a asumir retos para continuar con los procesos administrativos, de enseñanza, de aprendizaje y evaluativos. Lo anterior implica revisar roles, competencias, conocimientos, habilidades, y contenidos desde lo pedagógico y didáctico.

Actualmente, persisten aspectos críticos que hay que resolver de forma inmediata y otros de cara a la implementación de modelos de alternancia, para garantizar el normal desarrollo de los procesos educativos. En este sentido, es necesario revisar la organización escolar y el trabajo de los docentes y la comunidad educativa. Lo anterior, relacionado con el acceso a dispositivos tecnológicos y de conectividad, las competencias digitales de los docentes y estudiantes, de sus condiciones de vulnerabilidad, que exigen propuestas adaptadas y contextualizadas para responder a la necesidades de proceso educativo con calidad sin permitir brechas.

A nivel de programas de formación de docentes, al iniciar el primer semestre (mediados de febrero de 2020), los procesos de la práctica pedagógica se estaban desarrollando bajo lo planeado, pero la declaración de la crisis sanitaria y el aislamiento obligatorio por parte de los entes gubernamentales, llevo a las autoridades académicas a replantear dicho proceso, ya que, de manera inesperada, sin el conocimiento suficiente se hicieron adaptaciones a los mecanismos existentes con ayuda de las TIC para garantizar las tutorías y acompañamiento pedagógico de los docentes en formación en cada una de sus prácticas, lo que ocasionó desconcierto e incertidumbre frente a estos procesos (migrar de la educación presencial a la remota).
Afortunadamente, para el caso de la Licenciatura en Matemáticas de la Universidad Francisco de Paula Santander - Cúcuta, la mayoría de los docentes formadores había hecho uso de la plataforma PLAD (Portal de Apoyo a la Docencia - presencial, Prada-Núñez et al., 2019), y algunos tenían formación el uso de las TIC y ambientes virtuales de aprendizaje, pero era necesario y urgente pensar en otras formas de relación e interacción para garantizar el proceso de práctica pedagógica. Es claro que durante esta transformación había más interrogantes que respuestas, ya que tal vez lo único claro era necesidad de adaptar la práctica pedagógica presencial hacia una modalidad remota; situación que confirmó el rol primordial del docente formador ante el reto educativo para realizar el acompañamiento y asesoría de los docentes en formación y que debido a la flexibilización de la educación remota permitió hacer diversas adaptaciones a las prácticas pedagógicas en cuanto a destinatarios, intervención, objetivos, tiempos y espacios.

Por otra parte, hay que tener en cuenta la incertidumbre, consecuencia del comportamiento de cómo avanzará la pandemia, la falta de certeza de cuándo se regresará a la normalidad (que incluye volver a las clases presenciales), dando como resultado afectaciones muy graves para el aprendizaje de los estudiantes.

Ahora bien, desde el programas de Licenciatura en Matemáticas se hace necesario resignificar y transformar las prácticas pedagógicas de los docentes en formación. Por ello el objetivo de este estudio es describir el desarrollo de los procesos de práctica pedagógica durante la emergencia de la pandemia, ante la ausencia de información sobre los efectos que está teniendo en los de los docentes en formación, así como aportar elementos de autoevaluación para el desarrollo de las prácticas pedagógicas. 


\section{EDUCACIÓN REMOTA VS EDUCACIÓN VIRTUAL}

Pensar que la educación remota y la virtual son iguales sería un error técnico y metodológico. Es cierto que se pueda dar la confusión debido a que tienen características similares, pero sus diferencias son notorias. La educación remota, es una adaptación del sistema educativo presencial que se fundamenta en sistemas digitales donde se establecen tiempos de conexión, se parametriza un sistema de calificaciones que no es flexible y se dejan un sin número de trabajos, mientras que la educación virtual es personalizada, autónoma y cuenta con plataformas para la creación de contenidos basados en las necesidades de los aprendices, fundamentado en el aprendizaje colaborativo, lo que permite que docentes y estudiantes compartan de forma digital información y conocimiento (Pachón, 2021; García- Quintero \& Villamizar, 2017).

A raíz de la crisis del Covid 19, se habla de educación remota de emergencia, siendo esta un cambio temporal del proceso de enseñanza alternativa debido a circunstancias de crisis. El objetivo de esta modalidad es proporcionar acceso temporal a la enseñanza y a los apoyos de una manera rápida y fácil de instalar durante una emergencia o crisis (Hodges et al., 2020).

\section{METODOLOGÍA}

Esta investigación se enmarca en las características del enfoque cuantitativo a nivel descriptivo y de corte transversal, dado que los datos fueron recolectados durante las dos primeras semanas de iniciado el primer semestre del 2021 con el objetivo que los estudiantes evaluaran el proceso educativo adelantando en las asignaturas de Práctica Pedagógica durante la situación atípica de no presencialidad derivada por la declaratoria de pandemia ante el Covid-19 en nuestro país.
Para la recolección de los datos el grupo de investigadores diseñó un cuestionario ad hoc compuesto por 28 ítems con opciones de respuesta cerrada, en los que se pretendía determinar el impacto del proceso adelantado durante los dos semestres del 2020 en la línea de asignaturas denominadas Práctica Pedagógica, como parte del componente Pedagógico dentro del proceso de formación de los futuros Licenciados en Matemáticas. Para tal fin, después de ser aprobado el cuestionario por juicio de expertos, se procedió a crear un formulario por medio de Google Form, el cual posteriormente fue compartido a los estudiantes con una carta de invitación al diligenciamiento de éste, resaltando la importancia de este proceso dentro del proceso de autoevaluación y diseño de planes de mejora para la renovación de la Acreditación de Alta Calidad, reconocimiento que ostenta el programa actualmente.

La población objeto de investigación estaba conformada por la totalidad de estudiantes matriculados en el primer semestre del 2021 en el programa en mención, que corresponde a un tamaño de 289 estudiantes. De ellos se logró consolidar una muestra no probabilística de 68 estudiantes los cuales cumplieron los siguientes criterios de inclusión: a) haber cursado durante el año 2020 cualquiera de las siguientes asignaturas Práctica pedagógica en contextos, Práctica pedagógica en contextos especiales, Práctica profesional I y Práctica profesional II; y b) estar matriculado en el primer semestre del 2021. La selección de estas cuatro asignaturas corresponde al inicio y fin del proceso de formación de competencias pedagógicas.

Una vez los datos fueron recolectados, se procedió a descargar la data como un archivo de Excel, luego fue revisado en detalle para evitar inconsistencias en el ellos y finalmente se exportaron al software SPSS para ser procesados, organizar la información por medio de tablas, estadísticos o gráficos y así analizarla. 


\section{RESULTADOS Y DISCUSIÓN \\ PERFIL DEMOGRÁFICO DE LOS ENCUESTADOS}

Por medio de la tabla 1 se puede identificar que existe predominio del género masculino en la muestra de encuestados, observando que el $66.2 \%$ de ellos tienen edades que oscilan entre 18 y 23 años. Respecto a la distribución de ellos en función de la asignatura vista, se pudo observar en ambos semestres que aproximadamente el $60.0 \%$ de ellos se concentran en las primeras prácticas las cuales se ubican en el ciclo de Especialización dentro de la propuesta curricular, mientras que el porcentaje restante se concentra en las prácticas correspondientes al ciclo de Profundización.

Tabla 1. Características demográficas de los estudiantes

\begin{tabular}{|c|c|c|}
\hline Variable & Nivel de respuesta & Porcentaje \\
\hline \multirow{5}{*}{ Rango de edad } & De 18 a 20 años & $20.6 \%$ \\
\hline & De 21 a 23 años & $45.6 \%$ \\
\hline & De 24 a 26 años & $25.0 \%$ \\
\hline & 27 años o más & $8.8 \%$ \\
\hline & Total & $100.0 \%$ \\
\hline \multirow{3}{*}{ Género } & Femenino & $39.7 \%$ \\
\hline & Masculino & $60.3 \%$ \\
\hline & Total & $100.0 \%$ \\
\hline \multirow{5}{*}{$\begin{array}{l}\text { Práctica cursada en el } \\
\text { Primer semestre del } 2020\end{array}$} & Práctica Pedagógica en Contextos & $27.9 \%$ \\
\hline & Práctica Pedagógica en Contextos Especiales & $30.9 \%$ \\
\hline & Práctica Profesional I & $26.5 \%$ \\
\hline & Práctica Profesional II & $14.7 \%$ \\
\hline & Total & $100.0 \%$ \\
\hline \multirow{5}{*}{$\begin{array}{l}\text { Práctica cursada en el } \\
\text { Segundo semestre del } \\
2020\end{array}$} & Práctica Pedagógica en Contextos & $29.4 \%$ \\
\hline & Práctica Pedagógica en Contextos Especiales & $26.5 \%$ \\
\hline & Práctica Profesional I & $20.6 \%$ \\
\hline & Práctica Profesional II & $23.5 \%$ \\
\hline & Total & $100.0 \%$ \\
\hline
\end{tabular}

Fuente: Elaboración propia

\section{ESCENARIO DE TRABAJO}

Al indagar entre el grupo de estudiantes sobre si la práctica pedagógica la habían desarrollado en alguna institución educativa o si se había adelantado trabajo alternativo ante la declaratoria de aislamiento social, se determinó que durante el primer semestre del 2020 el $51.0 \%$ de los estudiantes no realizaron prácticas en instituciones educativas, afirman que con los docentes de la universidad se realizaron una serie de actividades sincrónicas y asincrónicas asistidas por tecnologías en las que se abordaron diversos temas en competencias pedagógicas pero sin ir estar vinculados a ninguna institución educativa. Esto se puede explicar en el hecho, que durante el mes de marzo se decreta el aislamiento social, inicialmente por un tiempo de 15 días, para luego ser prorrogado a 45 días y así sucesivamente hasta llegar al mes de junio sin que en las instituciones educativas se desarrollara actividad alguna. 


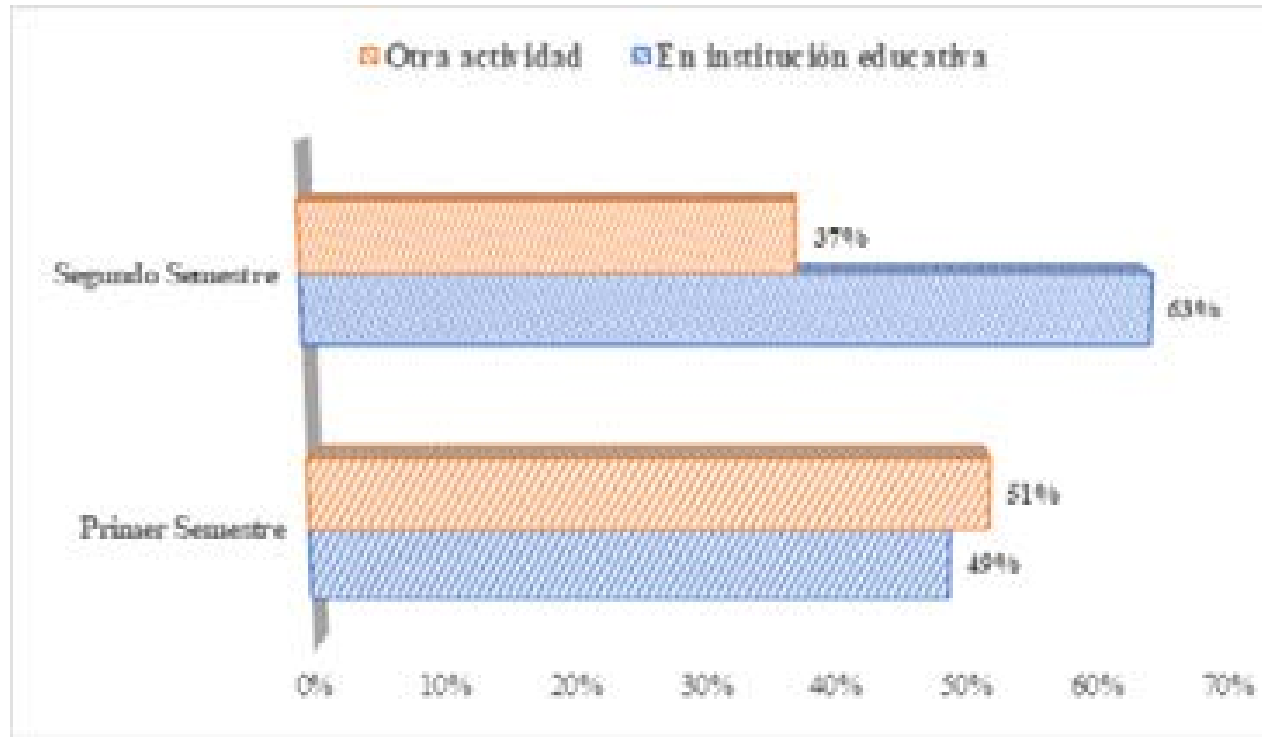

Figura 1. Comparativo de posibles escenarios de prácticas realizados durante el 2020

Ya para el segundo semestre del 2020, todos los docentes en formación matriculados desarrollaron su práctica pedagógica, esto debido a que los profesores de la universidad como en las instituciones educativas, acumulaban ciertas directrices de cómo adelantar el proceso pedagógico sin presencialidad, por ello el porcentaje de estudiantes en escenarios de práctica aumentó con respecto al semestre anterior. Aunque en la Figura 1 refleja que un $37 \%$ de los docentes en formación no asistieron a instituciones educativas, esto se debe a que en el VII semestre de su malla curricular los docentes en formación no realizan prácticas pedagógicas o se encontraban cursando la asignatura de Práctica Habilidades Pedagógicas.

\section{RECURSOS UTILIZADOS COMO ESTUDIANTE}

Se indagó inicialmente sobre la disponibilidad de recursos tecnológicos con los que cuentan los estudiantes para el desarrollo de sus actividades académicas, determinando que el $39.7 \%$ de ellos se habían conectado utilizando sus teléfonos inteligentes, mientras que el $58.8 \%$ accedían utilizando computador de mesa, seguido el $39.7 \%$ quienes lo hacen utilizan computador portátil, y el $1.5 \%$ restante aseguran no poseer ningún recurso tecnológico para el acceso a sus clases por lo que deben acudir a café internet $y$ en caso de no tener dinero para pagar el alquiler, pues simplemente no se conectaban a las clases.

De aquellos que afirmaron acceder por medio de su teléfono móvil, sólo el $8.8 \%$ lo hacían dentro del plan de datos contratado en modalidad pospago, el resto se conectan a internet por medio de redes Wifi, algunos a su red casera $(41.2 \%)$ mientras que el $47.1 \%$ accedían por medio de redes inalámbricas abiertas o de carácter público. 


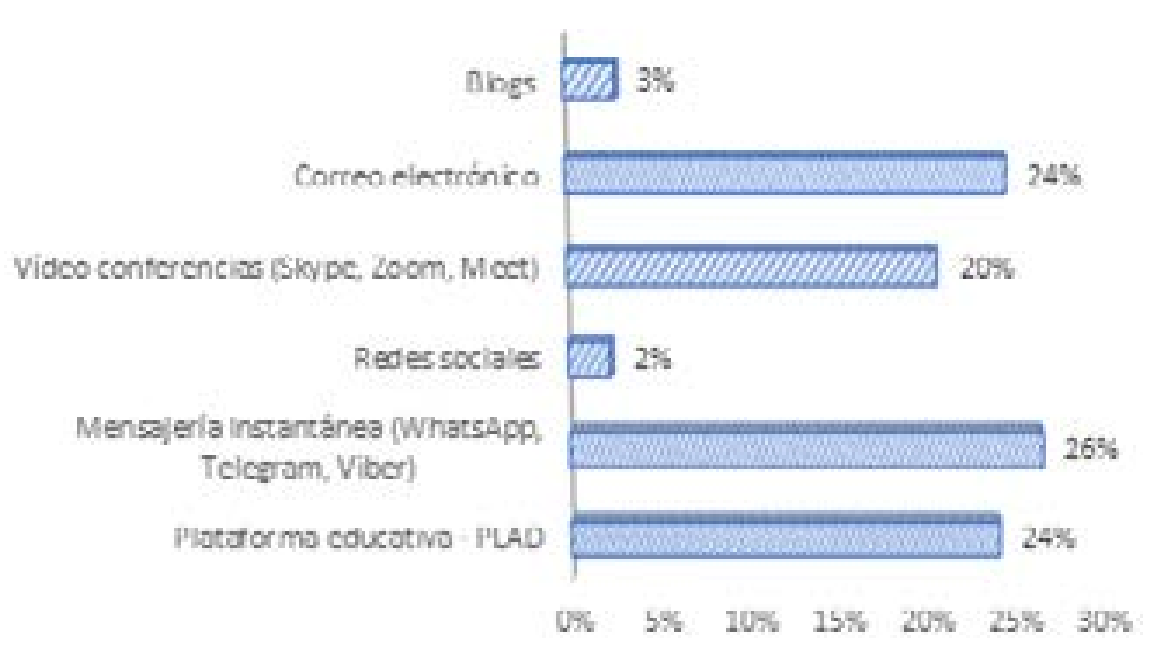

Figura 2. Recursos tecnológicos utilizados durante el 2020

Por medio de la figura 2, se pudo determinar el medio más utilizado para establecer comunicación entre los docentes y los estudiantes de la universidad, había sido la mensajería instantánea siendo el WhatsApp la aplicación más utilizada, le siguen con igual porcentaje el uso del correo electrónico y la plataforma institucional denominada PLAD (Prada-Núñez et al., 2019). Las aplicaciones para vídeo conferencias como Meet, Zoom o Skype ocupan el $20.0 \%$ de las opiniones y finalmente los blogs o redes sociales concentran en conjunto el $5.0 \%$ de las opiniones.

Finalmente, al indagar entre los estudiantes sobre el nivel de dominio que poseen sobre el manejo de los diversos recursos tecnológicos, se determinó que el $83.9 \%$ se consideran competentes, mientras que el $16.2 \%$ restante aseguran que requieren de diversos programas de capacitación o nivelación, para así hacer un uso adecuado de estos recursos.

\section{DESARROLLO DE SU PRÁCTICA PEDAGÓGICA}

Al explorar en los docentes en formación sobre el trabajo que realizaron con sus estudiantes en el desarrollo de su práctica pedagógica, se pudo determinar que el $86.8 \%$ de ellos aseguran que tuvieron algún tipo de dificultad durante el correr del semestre, principalmente propiciado por las dificultades de conectividad. Afirman, los docente en formación que la creación de grupos de WhatsApp (en el $85.3 \%$ de los casos) fue el principal medio de comunicación para el envío de material o para comunicarse con sus estudiantes (López-García \& Gutiérrez-Niño, 2018; Prada-Núñez et al., 2020; Espinel-Rubio et al., 2021).

Estos procesos de acompañamiento pedagógico los realizaban cumpliendo con el horario de clases, siendo al menos una vez por semana; dejando como evidencias del trabajo realizado principalmente el material de apoyo creado y que fue compartido a los estudiantes de las instituciones educativas, mientras que algunos fueron más allá dejando evidencias fotográficas de los estudiantes conectados (cuando se podía) o videos de los pocos espacios en dónde contaron con la presencia de estos.

El trabajo que ellos realizaron en las instituciones educativas contó en la mayoría de las veces con la supervisión de los docentes tanto de la universidad como de la institución educativa en donde adelantaban la práctica. Para el caso de las asesorías con los docentes de la universidad, 
predominó en el $57.4 \%$ de los casos la realización de video conferencias por Meet (Ayala-García et al., 2020), mientras que para el caso de los profesores de la institución educativa se recurrió principalmente a la comunicación vía WhatsApp y en algunos casos, llamadas telefónicas. Este proceso de acompañamiento se realizaba semanalmente y de forma grupal con el docente de la universidad, mientras que el docente de la institución educativa las realizaba de forma individual.

En todos los casos, los estudiantes que realizaron actividades en institución educativa lo hicieron por medio del uso de diversos recursos TIC. Como ya se mencionó los estudiantes de las instituciones educativas tenían dificultades de acceso a los recursos TIC, reportando que en el $65.2 \%$ de los casos, aproximadamente el $40.0 \%$ de los estudiantes de cada curso se podían conectar, por medio de computador de mesa en el $7.3 \%$ de los casos y el $63.2 \%$ lo hacían por medio del uso de un Smartphone, aspecto que limitaba el trabajo pedagógico.

Esta limitación de acceso a los recursos tecnológicos empezó a ser más evidente en la medida que en cada hogar los miembros empezaron a hacer un uso masivo de ellos, puesto que en muchos hogares sólo había un PC o un smartphone que debía compartirse entre los hijos y en algunos casos, con los padres.

\section{PERCEPCIÓN DEL NIVEL DE AFECTACIÓN DE LA EMERGENCIA SANITARIA}

Finalmente, se exploró en los estudiantes practicantes su opinión sobre las diversas afectaciones que se derivaron del aislamiento social y de la mediación y apropiación de las TIC para el desarrollo de procesos pedagógicos no presenciales. Los estudiantes encuestados opinan que:

- El $75.0 \%$ de los encuestados aseguran que los estudiantes se encontraban desmotivados en el desarrollo de los cursos, posiblemente propiciados por las limitaciones que tenían de acceso a los diversos recursos tecnológicos, lo cual se podría decir, que reafirmó las diferencias de brechas sociales.

- El $66.2 \%$ reconocen que esta situación produjo en ellos una larga lista de comportamientos de rechazo que terminaron desencadenando actitudes y comportamientos negativos hacia el proceso educativo.

- Finalmente, en el $77.9 \%$ de los casos, los docentes en formación aseguran que la pandemia les afecto de alguna forma el desarrollo de sus diversos cursos de práctica pedagógica debido a que los estudiantes a su cargo se encontraban desmotivados por las limitaciones que tenían de acceso a los diversos recursos tecnológicos.

\section{ESTRATEGIAS DE APROPIACIÓN DE LAS PRÁCTICAS PEDAGÓGICAS MEDIANTE LA EDUCACIÓN REMOTA CON TIC EN ÉPOCA DE PANDEMIA}

A partir del hecho que educación remota y virtual no son lo mismo y teniendo en cuenta que los procesos de practica pedagógica son presenciales para realizar la adaptación se toma como referente la implementación de la educación remota, pero apoyándose en algunos elementos de la educación virtual (Pachón, 2021; Hodges et al., 2020)

Para afrontar la situación excepcional generada por la crisis de la pandemia, se implementó un plan de contingencia apoyado en la educación remota, con algunos elementos de educación virtual, entendiendo las limitantes y posibilidades de los estudiantes, para conectarse o no a las tutorías y acompañamiento pedagógico, (el 
cual cambia por la mediación tecnológica), pero conserva su proceso misional.

Las estrategias de adaptación de la práctica pedagógicas mediante la educación remota con TIC se apoyan en:

- La mediación y apropiación de las TIC con finalidad pedagógica. Una práctica pedagógica mediada por las TIC debe ser comprendida como una evolución del proceso enseñanza-aprendizaje, que permite mediante la tecnología, abrir un espacio formativo a las nuevas opciones que dinamizan el espaciotiempo más allá de la presencialidad física. Esto es posible, si el docente tutor - supervisor de práctica entiende el sentido de la utilización de las tecnologías reconociendo utilidad de estas en las propias experiencias docentes de preparación de materiales, manejo de información digital, presentar contenidos o comunicarse con los docentes en formación (Núñez et al., 2019). En este sentido, el Ministerio de Educción Nacional (2013), estableció las Competencias TIC para el Desarrollo Profesional Docente que se constituyen en acuerdos conceptuales y lineamientos para orientar los procesos formativos en el uso pedagógico de las TIC y que son aplicables a los procesos de practica pedagógica para la formación docentes (Hernández-Suárez et al., 2016).

- La tutoría y acompañamiento pedagógico. La tutoría es un proceso orientador en el que el profesor-tutor $y$ el estudiante se encuentran en un espacio común (en este caso virtual), con un marco teórico referencial y una planificación previa, el primero ayude al segundo en aspecto académicos, profesionales, sociales y personales, y juntos puedan diseñar su plan, en este caso de la práctica pedagógica (Giner et al., 2013). Por otra parte, el acompañamiento pedagógico, es un proceso sistemático y permanente, mediado por el docente tutor supervisor de práctica, con el objeto de interactuar con los docentes en formación para promover la reflexión sobre su actividad docente, toma de decisiones para realizar los cambios necesarios y gestión como el conjunto de acciones que permiten concebir, planificar, organizar, ejecutar y controlar de manera sistemática y permanente el perfeccionamiento del desempeño docente, a través de interacciones pedagógicas de carácter profesional, interpersonal y de contexto que, en correspondencia con las necesidades, requerimientos y características de los docentes en formación potencien los saberes necesarios para transformar la práctica y alcanzar los objetivos esperados, garantizar el logro de aprendizajes en una perspectiva integral (Díaz et al., 2018).

- El trabajo pedagógico colaborativo, que se refiere a la asociación de varias personas para la práctica de la participación, observación, corrección y esfuerzo, describiendo un rol para cada miembro del grupo, pero que se relacionan, complementan y diferencian en consecución de una meta común (Orozco, 2011).

\section{CONCLUSIÓN}

Como conclusión, y teniendo en cuenta que el cierre de las instituciones educativas en todos los niveles educativos, por la pandemia del Covid-19, obligó a crear nuevas formas de interacción entre docentes, estudiantes y comunidad educativa en general, lo que condicionó el desarrollo de 
las prácticas pedagógicas de forma presencial, pero posibilitó otras formas para continuar con el desarrollo de las prácticas pedagógicas con el diseño e implementación de estrategias para el acompañamiento de los docentes en formación apoyado principalmente en la educación remota con TIC. Esto involucró cambios a nivel metodológico (tiempos, espacios, estrategias y el contexto), el reevaluar contenidos y objetos de enseñanza - aprendizaje que permitieron la mediación las TIC a los procesos formativos de la práctica pedagógica.

\section{REFERENCIAS}

Ayala-García, E., Hernández-Suárez, C., \& Prada-Núñez, R. (2020). Proceso educativo en programas de Arquitectura bajo el aislamiento preventivo obligatorio por causa del COVID-19. Educación y Humanismo, 22(39), 1-25. https://doi. org/10.17081/eduhum.22.39.4205

Barrutia-Barreto, I., Sánchez-Sánchez, R. M., \& Silva, H. A. (2021). Consecuencias económicas y sociales de la inamovilidad humana bajo Covid - 19 caso de estudio Perú. Lecturas De Economía, (94), 285303. https://doi.org/10.17533/udea. le.n94a344397

Díaz, M., García, J., \& Legañoa, M. Á. (2018). Modelo de gestión del acompañamiento pedagógico para maestro de primaria. Transformación, 14(1), 44-57. https:// revistas.reduc.edu.cu/index.php/ transformacion/article/view/1926

Espinel-Rubio, G. A., Hernández-Suárez, C. A., \& Prada-Núñez, R. (2021). Usos y gratificaciones de las redes sociales en adolescentes de educación secundaria y media: de lo entretenido a lo educativo. Encuentros, 19(01). https:// doi.org/10.15665/encuen.v19i01.2552
Gamboa-Suárez, A. A., Hernández-Suárez, C. A., \& Prada-Núñez, R. (2020). Efectos depresivos del aislamiento preventivo obligatorio asociados a la pandemia del Covid-19 en docentes y estudiantes de una universidad pública en Colombia. Psicogente, 24(45), 1-20. https://doi. org/10.17081/psico.24.45.4156

García-Quintero, C., \& Villamizar Suárez, G. (2017). Análisis fenomenológico de la conciencia del docente a partir de sus prácticas evaluativas. Revista Perspectivas, 2(2), 49-59. https://doi. org/10.22463/25909215.1313

Giner, Y., Muriel, M. J., \& Toledano, F. J. (2013). De la tutoría presencial a la virtual: la evolución del proceso de tutorización. REDU. Revista de Docencia Universitaria, 11(2), 89-106. http://red-u. net/redu/files/journals/1/articles/553/ public/553-2367-1-PB.pdf

Hernández-Suárez, C., Arévalo-Duarte, M., \& Gamboa-Suárez, A. (2016). Competencias TIC para el desarrollo profesional docente en educación básica. Praxis \& Saber, 7(14), 41 - 69. https://doi.org/10.19053/22160159.5217

Hernández-Vergel, V. K., Prada-Núñez, R., \& Hernández-Suárez, C. A. (2021). Afectaciones ocupacionales y emocionales derivadas del aislamiento social en tiempos del Covid-19. Revista Boletín Redipe, 10(2), 295-311. https:// doi.org/10.36260/rbr.v10i2.1214

Hodges, C., Moore, S., Lockee, B., Trust, T., \& Bond, A. (27 de marzo de 2020). The Difference Between Emergency Remote Teaching and Online Learning. EDUCAUSE Review. https:// 
er.educause.edu/articles/2020/3/thedifference-between-emergency-remoteteaching-and-online-learning

López-García, J. D., \& Gutiérrez-Niño, D. (2018). Efecto del uso de la herramienta "realidad aumentada" en el rendimiento académico de estudiantes de Educación Básica. Revista Perspectivas, 3(1), 6-12. https://doi.org/10.22463/25909215.1464

Ministerio de Educación Nacional. (2013). Competencias TIC para el desarrollo profesional docente. Bogotá: Mineducación. https:// www.mineducacion.gov.co/1759/ articles-339097 archivo pdf competencias tic.pdf

Núñez, C., Gaviria-Serrano, J. M., Tobón, S., Guzmán-Calderón, C. E., \& Herrera, S. R. (2019). La práctica docente mediada por TIC: una construcción de significados. Espacios, 40(5), 1-15. https://revistaespacios.com/a19v40n05/ a19v40n05p04.pdf

Orozco, R. (2011). El trabajo pedagógico colaborativo como metodología para la formación profesional durante la Práctica Supervisada. InterSedes, 6(11), 57-71. https://revistas.ucr.ac.cr/ index.php/intersedes/article/view/951

Pachón, J. (2021). La educación remota no es sinónimo de educación virtual. Revista educación virtual. https://revistaeducacionvirtual. com/archives/3462\#: : :text=La\%20 educaci $\%$ C3\%B3n $\% 20$ remota $\% 20$ es $\% 20$ tan,virtual $\% 20$ crea $\% 20$ $1 \%$ C3\%ADderes $\% 20 y \% 20$ mentores.

Prada Núñez, R., Hernández Suárez, C. A. y Gamboa, A. A. (2019). Usos y efectos de la implementación de una plataforma digital en el proceso de enseñanza de futuros docentes en matemáticas. Revista Virtual Universidad Católica del Norte, (57), 137-156. https://doi. org/10.35575/rvucn.n57a10

Prada-Núñez, R., Ayala-García, E. T., \& Hernández-Suárez, C. A. (2020). Modelación matemática de las afectaciones fisiológicas en la comunidad académica en respuesta al Covid-19. Espacios, 41(42), 234247. https://doi.org/10.48082/espaciosa20v41n42p20

Prada-Núñez, R., Hernández-Suárez, C. A., \& Maldonado-Estévez, E. A. (2020). Diagnóstico del potencial de las redes sociales como recurso didáctico en el proceso de enseñanza en época de aislamiento social. Revista Espacios, 41(42), 260-268. https://doi. org/10.48082/espacios-a20v41n42p22 